\title{
Comparison of Ethanol and Acetone Mixtures for Extraction of Condensed Tannin from Grape Skin
}

\author{
M.O. Downey*, R.L. Hanlin \\ Department of Primary Industries Victoria, PO Box 905, Mildura, VIC 3502, Australia \\ Submitted for publication: April 2010 \\ Accepted for publication: September 2010 \\ Key words: Condensed tannin, extraction, ethanol, acetone, phloroglucinolysis, HPLC
}

\begin{abstract}
Discrepancies in condensed tannin concentrations in grape skin determined by different analytical methods prompted the closer examination of aspects of the methodologies. One of these was the choice of extraction solvent. Condensed tannins were extracted from Shiraz grape skins using a range of aqueous solvent mixtures ranging from zero to $100 \%$ acetone and ethanol to examine the relative effectiveness of each solvent mixture and to determine whether different solvent mixtures extracted similar condensed tannin components. Acetone extracted more condensed tannin than ethanol. Mixtures of 50 to $\mathbf{7 0} \%$ acetone were equally effective. The most effective ethanol concentration was 50\%. Epicatechin-gallate terminal subunits were not detected by HPLC following acid-catalysed cleavage in any of the extraction solvents. Extension subunit composition was similar between solvents across most mixtures. Polymers were generally shorter in the ethanol extracts than in the acetone extracts. Despite differences in tannin concentration and polymer length, the subunit composition was similar in $50 \%$ ethanol and $70 \%$ acetone. More tannin and tannins with longer polymer lengths were extracted with $70 \%$ acetone than with $50 \%$ ethanol. This suggests that all grape skin tannins are similar in composition, varying only in length. Thus, $50 \%$ ethanol and $70 \%$ acetone would give a fair indication of the grape skin tannin composition extracted into wine. However, both $50 \%$ ethanol and $\mathbf{7 0 \%}$ acetone may overestimate the amount of tannin that is extracted into wine, as wine typically has a much lower solvent concentration, ranging between 10 and $15 \%$ ethanol.
\end{abstract}

\section{INTRODUCTION}

Wine grape tannins are significant contributors to wine quality (Gawel, 1998). The measurement of tannins in wine grapes before harvest gives an indication of the tannins that could be extracted into the wine during winemaking, and of the quality of the wine (Skogerson et al., 2007). There are a number of published methods for assessing tannin levels in grapes, ranging from the precipitation of tannins by protein (BSA) or methylcellulose (Harbertson et al., 2002; Sarneckis et al., 2006) to analysis by high-performance liquid chromatography (HPLC) (Kennedy \& Jones, 2001; Peng et al., 2001). These approaches require the extraction of the condensed tannins from the grape tissue prior to analysis, regardless of the analytical method.

A number of extraction solvents have been trialled, including ethanol, methanol, acetone, n-butanol, diethyl ether, ethyl acetate and aqueous and/or acidified mixtures (Hagerman, 1988; Kantz \& Singleton, 1990; Alonso et al., 1991; Kallithraka et al., 1995; Pekic et al., 1998; Kelm et al., 2006). Numerous reports comparing a range of different extraction solvents populate the published literature (Alonso et al., 1991; Kallithraka et al., 1995; Pekic et al., 1998). However, much of the published research has only examined selected solvent mixtures rather than the full range of possible mixtures (Alonso et al., 1991; Kallithraka et al., 1995). Consequently, the effectiveness of different acetonewater and ethanol-water mixtures in extracting condensed tannin, particularly from grape skin, has not been compared.
The most commonly used solvent for proanthocyanidin extraction from grape berries is $70 \%$ acetone in water $(\mathrm{v} / \mathrm{v})$, although $50 \%$ ethanol in water (v/v) has also been used (Sarneckis et al., 2006; Mercurio et al., 2007). Recently, published research comparing different analytical techniques for determining condensed tannin concentrations in grape skin reported significant discrepancies between analytical methods (Seddon \& Downey, 2008; Harbertson \& Downey, 2009). One of the suggested causes of this difference was the extraction solvent employed. One method used 50\% aqueous ethanol (v/v), while the other used $70 \%$ acetone in water $(\mathrm{v} / \mathrm{v})$. The authors postulated that the different extraction solvents may have preferentially extracted different classes of the heterogeneous group of compounds that comprise condensed tannins.

The aim of this study was to compare the extraction efficiency of a range of aqueous mixtures of both acetone and ethanol as solvents for the extraction of condensed tannins from Shiraz grape skin. The compositional analysis of the tannin polymers extracted in each solvent mixture is also reported.

\section{MATERIALS AND METHODS}

\section{Chemicals}

Methanol, ethanol, formic acid, glacial acetic acid and hydrochloric acid (30\%) were purchased from Merck (Darmstadt, Germany). Phloroglucinol, sodium acetate, L-ascorbic acid, (+)-catechin, (-)-epicatechin and (-)-epicatechin gallate were purchased from

*Corresponding author: mark.downey@dpi.vic.gov.au [Fax: +61350514523]

Acknowledgements: The authors gratefully acknowledge the technical assistance of Dale Unwin (Department of Primary Industries, Victoria) and Abdu Ebrahim (Victoria University) in the preparation and processing of samples. This research was supported by the Victorian Department of Primary Industries and by the grape growers and winemakers of Australia through their investment body, the Grape and Wine Research and Development Corporation. 
Sigma-Aldrich (St. Louis, USA). Deionised water was obtained through a Millipore system supplied by Milli-RX AnalyticalGrade Water Purification System (Millipore, Billerica, USA).

\section{Grape samples}

Samples were collected during the 2009 vintage from the Deakin Estate vineyards in north-western Victoria (Australia; 34 $27^{\circ}$ South, $142^{\circ} 19^{\prime}$ East). Approximately 40 whole bunches were collected during commercial harvest and stored overnight at $4^{\circ} \mathrm{C}$ to remove field heat. Grape berries were removed from the rachis, and a sub-sample of the bulked berries was assessed for berry weight and skin weight. Grape skins were collected from the remainder of the berries by expulsion of the seeds and flesh. Skins were immediately frozen in liquid nitrogen and ground to a fine powder using an IKA grinder (A11 Basic grinder, IKA Works, Petaling Jaya, Malaysia). Samples were stored at $-80^{\circ} \mathrm{C}$ until required for analysis.

\section{Extraction and analysis of condensed tannins}

Aqueous mixtures of ethanol and acetone (0-100\% ethanol or acetone) were used to extract condensed tannins from the grape skin to determine the effectiveness of the solvents. Shiraz grape skin $(0.40 \mathrm{~g})$ were suspended in $4.0 \mathrm{~mL}$ of solvent, mixed briefly on a VM1 vortex mixer (Ratek Instruments, Boronia, Australia), sonicated for 20 minutes at room temperature (ca. $23^{\circ} \mathrm{C}$ ) using a FXP14D sonicator (Unisonics Australia, Sydney, Australia) and vortexed again. The samples were centrifuged for 5 minutes at $16110 \times \mathrm{g}$, using a Rotofix 32 A centrifuge (Hettich, Tuttlingen, Germany).

Three $100 \mu \mathrm{L}$ aliquots of the supernatant were transferred to fresh microfuge tubes and dried under vacuum at room temperature. Samples were suspended in $50 \mu \mathrm{L}$ phloroglucinolysis reagent and incubated at $50^{\circ} \mathrm{C}$ for $20 \mathrm{~min}$. The samples were quenched with sodium acetate $(50 \mu \mathrm{L})$ as described by Kennedy and Jones (2001). For each replicate, the $100 \mu \mathrm{L}$ reaction product was transferred to a HPLC autosampler vial. Reaction products were separated on two Phenomenex (Lane Cove, Australia) Onyx monolithic $\mathrm{C} 18$ columns $(100 \times 4.6 \mathrm{~mm}, 2 \mu \mathrm{m})$ by HPLC and their identities confirmed by retention times and mass spectral analysis according to the method described by Hanlin and Downey (2009). Terminal subunits of catechin, epicatechin and epicatechin-gallate were quantified against calibration curves of their corresponding pure standards, while extension subunits were quantified using a catechin calibration curve, followed by conversion to their individual subunit equivalents using published conversion factors for the extension subunit phloroglucinol adducts (Kennedy \& Jones, 2001). Total tannin was determined as the sum of the quantified subunits. Average polymer length was calculated as the sum of the known terminal and extension subunits divided by the terminal subunits (Guyot et al., 1999). Because average polymer length or degree of polymerisation (DP) offers only limited information on polymer length, and many combinations of terminal and extension subunit concentrations could result in the same average polymer length, we have refined the notation for polymer length to include the ratio of the terminal subunit concentration to the extension subunit concentration, with average polymer length reported in parentheses: [terminal:extension (average)]. This notation conveys all of the elements of polymer length, and terminal subunit concentration can be used as a surrogate for polymer number.

\section{RESULTS AND DISCUSSION}

\section{Total tannin}

The total condensed tannin subunits extracted using ethanol-water and acetone-water mixtures were determined by summation of the subunits after conversion to individual subunit concentrations (Fig. 1). Mixtures containing acetone were more effective at extracting

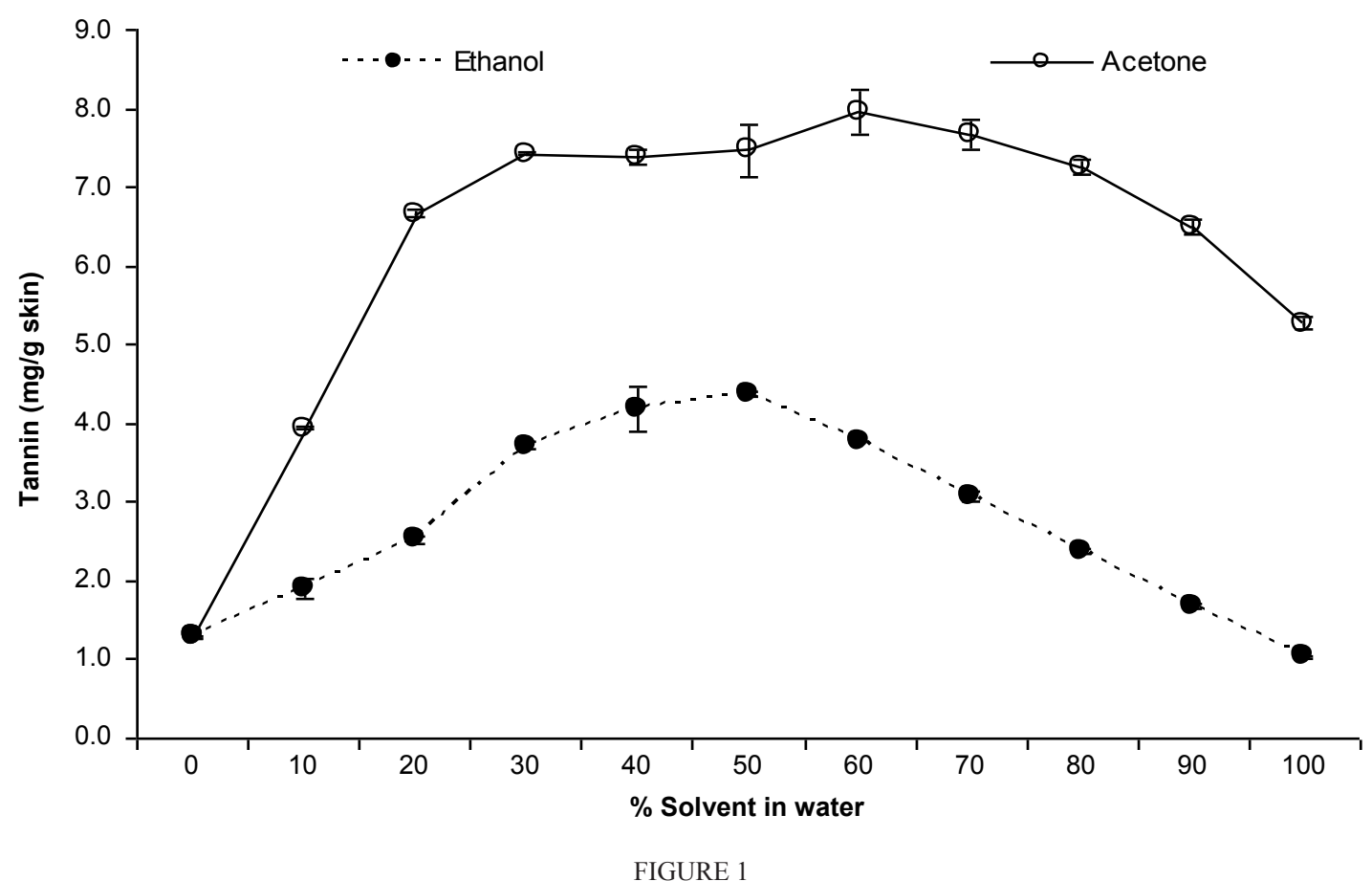

Concentration of total tannin extracted by different extraction solvents comprised of mixtures of ethanol and acetone in water. Data represents mean $+/-\mathrm{SE}(\mathrm{n}=3)$. 
tannins than those containing ethanol at a given concentration. Total tannin extracted from Shiraz grape skin increased with increasing proportions of ethanol, until a maximum extraction was reached at $50 \%$ ethanol in water. The concentration of extracted tannin then decreased in mixtures higher than $50 \%$ ethanol in water. The concentration of the extracted tannin was lowest in $100 \%$ ethanol and $100 \%$ water.

The concentration of extracted tannin increased steadily from zero percent acetone (100\% water). A maximum tannin concentration was reached between 50 and $70 \%$ acetone, and fewer condensed tannins were extracted above $70 \%$ acetone in water. At $100 \%$ acetone the level was greater than for $100 \%$ water, and greater than the amount extracted in 50\% ethanol. These observations were consistent with previously published research on total tannin extraction in ethanol and acetone (Kallithraka et al., 1995; Pekic et al., 1998; Harbertson \& Downey, 2009).

\section{Tannin polymer subunit composition}

Tannin polymer subunit composition was determined following the acid-catalysed cleavage of the polymer in the presence of phloroglucinol, i.e. a strong nucleophile reagent. Individual subunits were separated by reversed-phase HPLC, quantified against pure standards of catechin, epicatechin or epicatechingallate or converted to individual subunit equivalents using published conversion factors (Kennedy \& Jones, 2001).

An analysis of the terminal subunits, using various ethanol-water and acetone-water mixtures, revealed that the composition of the terminal subunits extracted in ethanol and acetone was similar for all mixtures (Fig. 2). Catechin and epicatechin terminal subunits were detected in the sample. Epicatechin-gallate terminal subunits were not detected. In ethanol, the proportion of catechin terminal subunits ranged from 59 to $66 \%$ of the total terminal subunits, while in acetone the proportion of catechin terminal subunits ranged from 63 to $70 \%$.

The absence of epicatechin-gallate as a terminal subunit in this sample was partly due to low levels observed in Shiraz skin from this site (Hanlin \& Downey, 2009), and partly related to the difficulty in identifying epicatechin-gallate at low levels in the background of unidentified peaks in the HPLC separation following phloroglucinolysis (Kennedy \& Jones, 2001; Gu et al., 2002).

These unidentified peaks are artefacts of the process of acidcatalysed cleavage in the presence of the nucleophile, which results in a range of nucleophile adducts and other products. The response of catechin and epicatechin standards to phloroglucinolysis was examined. Standards of catechin and epicatechin analysed by phloroglucinolysis and HPLC recovered $81.6 \%$ catechin and $84.5 \%$ epicatechin. Mass spectral analysis of the unidentified peaks present in the HPLC separation identified a $415 \mathrm{~m} / \mathrm{z}$ ion. This ion was consistent with previous reports that identified $415 \mathrm{~m} / \mathrm{z}$ as a flavan-3-ol adduct of phloroglucinolysis (Foo \& Karchesy, 1989). Mass spectral analysis also indicated the epimerisation of catechin to epicatechin and catechin to epicatechin. The epimerisation of flavan-3-ol monomers has also been observed in samples that have undergone thiolysis (Gu et al., 2002).

Extension subunit composition was similar for all mixtures of ethanol and acetone with water. Epigallocatechin and epicatechin
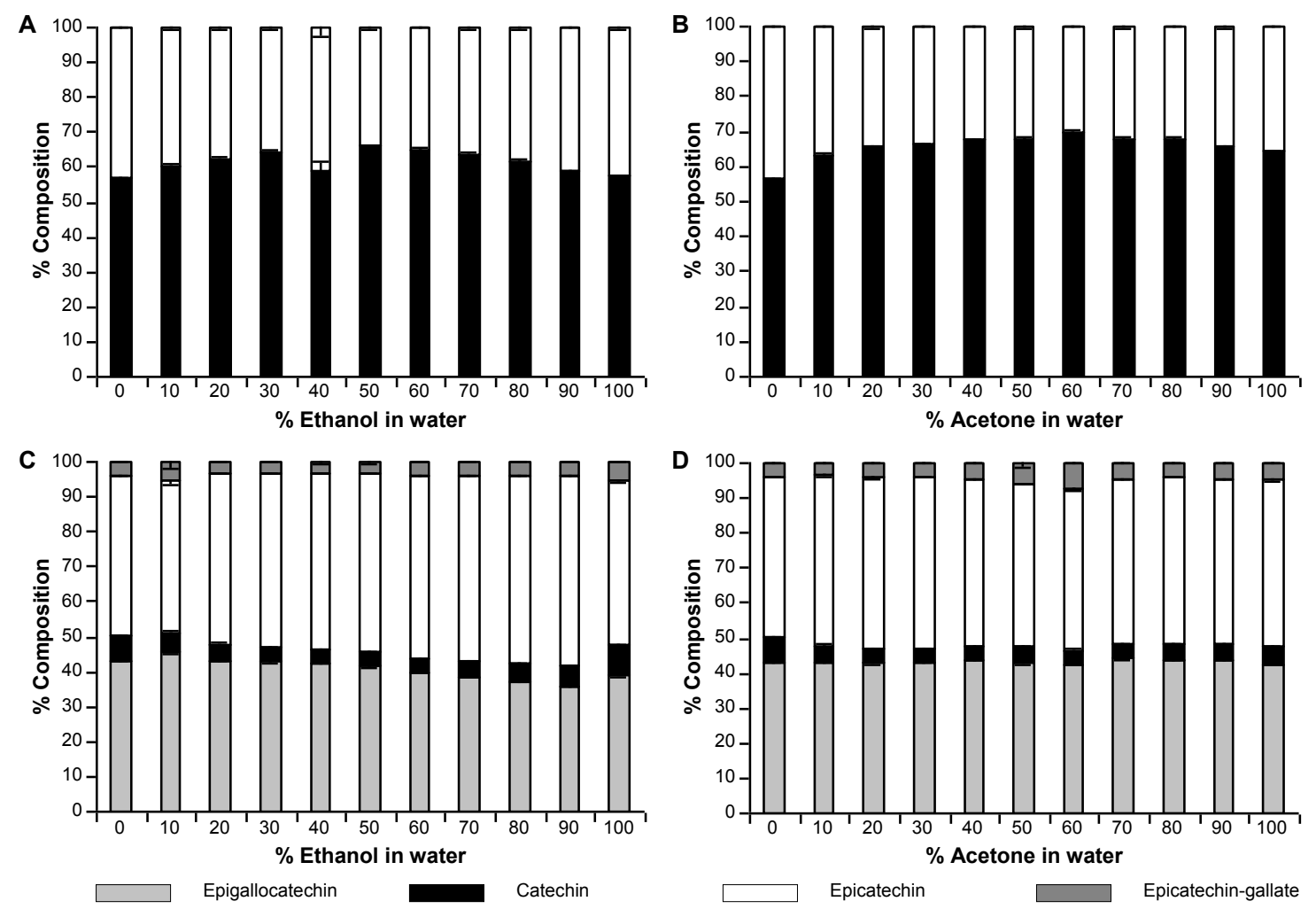

FIGURE 2

Proportional subunit composition of A) terminal subunits extracted in ethanol, B) terminal subunits extracted in acetone, C) extension subunits extracted in ethanol, D) extension subunits extracted in acetone. Data presented represents mean $+/-\mathrm{SE}(\mathrm{n}=3)$. 
in acetone-water mixtures represented around $45 \%$ of the total extension subunits each, while catechin and epicatechin-gallate represented around 5\% each, with some slight variation around these values (Fig. 2). The proportional composition of condensed tannin extension subunits was consistent with previously published data for Shiraz skin extraction (Downey et al., 2003; Hanlin \& Downey, 2009).

The extension subunit composition was virtually identical for zero and $10 \%$ ethanol, and similar between 20 and 50\% ethanol. The proportion of epigallocatechin extension subunits decreased and the proportion of epicatechin increased with an ethanol mixture higher than $50 \%$. The proportion of catechin and epicatechingallate extension subunits was relatively constant across all ethanol-water mixtures. The proportion of epicatechin ranged from $43.4 \%$ in $10 \%$ ethanol to $54.5 \%$ of extension subunits in $90 \%$ ethanol. Epigallocatechin ranged from 45.6 in $10 \%$ ethanol to $35.7 \%$ in $90 \%$ ethanol. Catechin extension subunits ranged from 4.0 to $8.6 \%$, while epicatechin-gallate extension subunits ranged from 3.1 to $5.4 \%$.

Epicatechin extension subunits ranged from $45.8 \%$ in $60 \%$ acetone to $48.8 \%$ in $30 \%$ acetone, while epigallocatechin extension subunits ranged from $42.6 \%$ in $60 \%$ acetone to $44.1 \%$ in $70 \%$ acetone. Catechin extension subunits ranged from 4.1 to $5.0 \%$, and epicatechin-gallate ranged from 3.6 to $4.7 \%$.

Small differences in the proportional composition of the condensed tannins extracted from Shiraz grape skin across a range of both acetone and ethanol mixtures suggest that both acetone and ethanol are extracting the same class of tannins. The other suggestion is that all tannins in grape skin are relatively similar and do not contain different subclasses of tannin. The main extension subunits were epicatechin and epigallocatechin in all the mixtures, with catechin and epicatechin-gallate extension subunits present at relatively low levels. These findings are consistent with previously published reports for Shiraz (Hanlin \& Downey, 2009).

The similarity in the proportional subunit composition of tannin polymers in all mixtures of acetone and ethanol signifies that both common extraction solvents, $50 \%$ ethanol and $70 \%$ acetone, are representative of the tannins extracted into wine, which is between 10 and $20 \%$ ethanol. However, the composition of tannin extracted into wine varies depending on the interactions of tannin with other wine components, such as yeast and cell wall metabolites that can bind tannin and prevent its extraction (Hazak et al., 2005). Winemaking practices can also influence wine tannin composition, for example through the addition of fining agents such as gelatine that preferentially removes tannin high in epicatechin gallate (Maury et al., 2003).

\section{Tannin polymer length}

The average polymer length ranged from 6.6 subunits in the water extraction to a maximum of 27.2 subunits in $60 \%$ acetone (v/v) and 17.0 subunits in $50 \%$ ethanol (Fig. 3). The average length of the tannin polymers extracted was significantly lower $(p<0.05$, $\mathrm{n}=3$ ) in all the ethanol mixtures than in the acetone mixtures. The average polymer length increased steadily in ethanol mixtures of $10 \%$ to $50 \%$ ethanol in water $(\mathrm{v} / \mathrm{v})$. A decrease in polymer length occurred with ethanol mixtures higher than $50 \%$ in water $(\mathrm{v} / \mathrm{v})$. At $100 \%$ ethanol, the average polymer length was 5.2 subunits, which was lower than the level extracted in $100 \%$ water (6.6 subunits). For the acetone mixtures there was no significant difference in the average polymer length of the tannins extracted in 30\% acetone $(\mathrm{v} / \mathrm{v})$ up to $80 \%$ acetone $(\mathrm{p}>0.05 ; \mathrm{n}=3)$.

If we examine the elements of average polymer length, i.e. the number of terminal subunits and average polymer length using

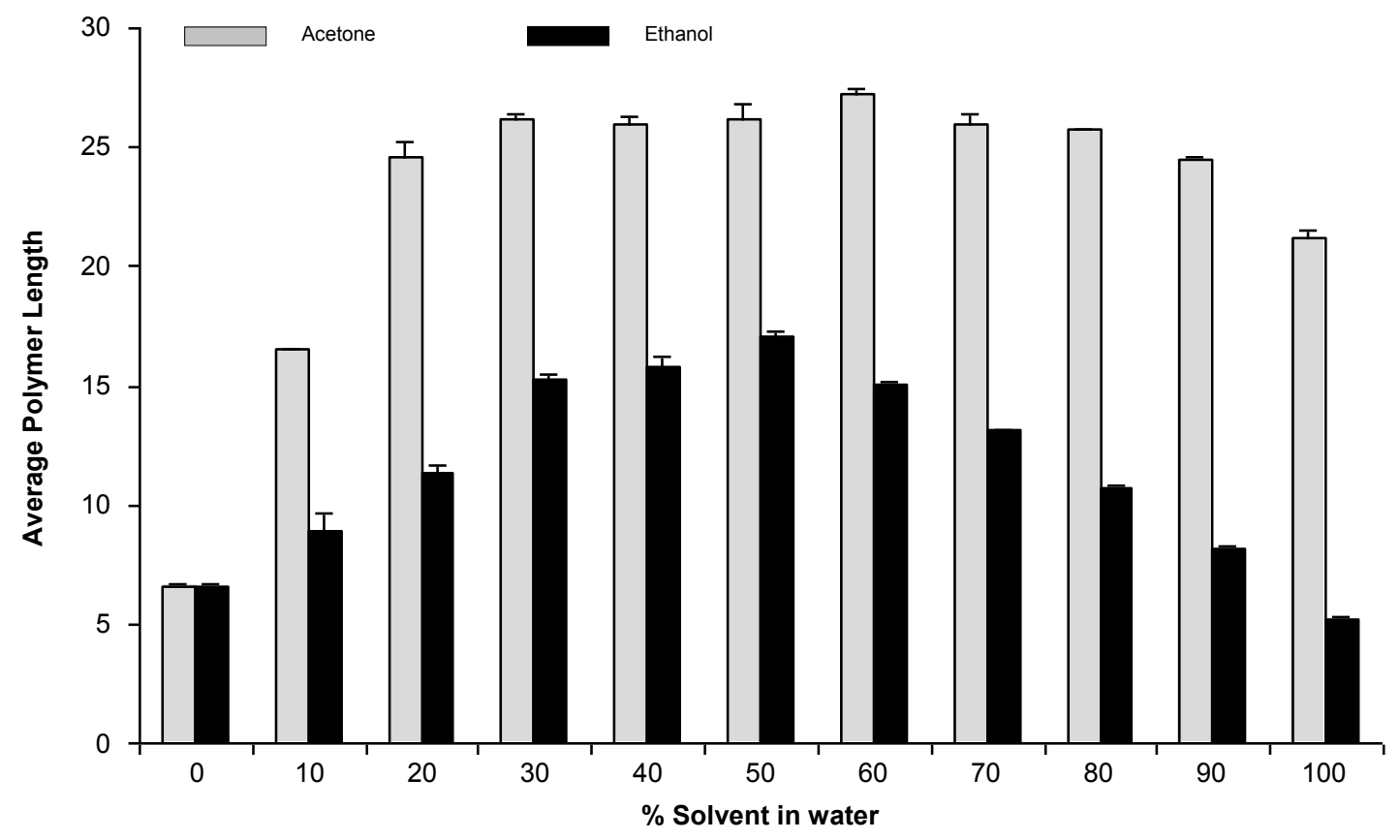

FIGURE 3

Average polymer length of condensed tannins extracted in aqueous mixtures of acetone (grey) and ethanol (black). Values represent mean $+/-$ SE $(n=3)$. 
the notation described above [terminal:extension (average)] (Table 1), it is apparent that the actual number of polymers, as represented by the concentration of terminal subunits, increased in the ethanol-water mixtures. Polymer number increased from zero to $40 \%$ ethanol and then declined in mixtures above $50 \%$ ethanol. Similarly, terminal subunit concentration, and thus polymer number, increased in acetone extracts from zero to $70 \%$ acetone and then decreased. The presentation of the data using the abovementioned notation shows that polymer number and average polymer length are greater in aqueous acetone extracts than in aqueous ethanol extracts.

While the terminal and extension subunit composition was relatively constant across most of the ethanol and acetone mixtures, average polymer length and polymer number were not. Thus, for analyses requiring a measure of total tannin in grape skin, 70\% acetone is the most effective solvent. However, for a winemaker the total amount of tannin in the grape skin is less important than the amount likely to be extracted during winemaking. Under these circumstances, $70 \%$ acetone would certainly overestimate the amount of tannin extracted and the length of polymers that might reasonably be expected in the fermenting wine.

Similarly, tannin extracted in $50 \%$ ethanol would also not be representative of the tannin extracted into wine. Wine is typically between 10 and $20 \%$ ethanol. It is interesting that, at 10 to $20 \%$ ethanol, average polymer length (Fig. 3) was between five and 10 subunits, similar to the polymer lengths in wine reported elsewhere (Monagas et al., 2003; Pastor del Rio \& Kennedy, 2006). This suggests that only small tannin polymers are extracted during winemaking, with large polymers remaining in the grape tissue, possibly bound to the cell wall matrix (Hanlin et al., 2010). For this reason, perhaps extraction with a model wine would be more appropriate for estimating potential grape skin tannin extraction into wine.

\section{TABLE 1}

Polymer length of condensed tannins extracted in aqueous mixtures of ethanol and acetone showing ratio of terminal to extension subunits with average polymer length expressed in parentheses - terminal:extension (average). Terminal and extension subunits expressed as concentration (mg/g fwt skin).

\begin{tabular}{ccc}
\hline$\%$ & Ethanol & Acetone \\
\hline $\mathbf{0}$ & $0.20: 0.98(6.6)$ & $0.20: 1.10(6.6)$ \\
$\mathbf{1 0}$ & $0.21: 1.68(8.9)$ & $0.24: 3.70(16.5)$ \\
$\mathbf{2 0}$ & $0.22: 2.30(11.3)$ & $0.27: 6.40(24.6)$ \\
$\mathbf{3 0}$ & $0.24: 3.48(15.3)$ & $0.28: 7.14(26.2)$ \\
$\mathbf{4 0}$ & $0.26: 3.92(15.8)$ & $0.28: 7.10(25.9)$ \\
$\mathbf{5 0}$ & $0.26: 4.11(17.0)$ & $0.29: 7.18(26.1)$ \\
$\mathbf{6 0}$ & $0.25: 3.52(15.1)$ & $0.29: 7.67(27.2)$ \\
$\mathbf{7 0}$ & $0.24: 2.85(13.1)$ & $0.30: 7.37(26.0)$ \\
$\mathbf{8 0}$ & $0.22: 2.16(10.7)$ & $0.28: 6.99(25.7)$ \\
$\mathbf{9 0}$ & $0.20: 1.47(8.2)$ & $0.27: 6.24(24.4)$ \\
$\mathbf{1 0 0}$ & $0.20: 0.84(5.2)$ & $0.25: 5.03(21.3)$ \\
\hline
\end{tabular}

As employed in this investigation and elsewhere, the method of tannin extraction, phloroglucinolysis and HPLC separation underestimates average polymer length because free monomeric flavan-3-ols are extracted with each of the solvent mixtures used. During HPLC separation, free monomers and terminal subunits are indistinguishable. To overcome this, some practitioners have determined free monomers separately and subtracted the concentration of free monomers from the total of free monomers and terminal subunits following HPLC-phloroglucinolysis. However, as discussed above, around $15 \%$ of monomeric flavan3-ols are converted to phloroglucinol adducts of flavan-3-ol degradation products during phloroglucinolysis. Thus, the total of free monomers and terminal subunits would be underestimated, which would overestimate average polymer length. This would also explain why some workers have observed very low, zero or sub-zero terminal subunit concentrations as a result of subtracting the independently determined free monomer concentration from the total of free monomers and terminal subunits following phloroglucinolysis. To overcome this limitation, in our laboratories we have employed liquid-liquid purification of the tannin extract to remove monomers and low molecular weight material prior to phloroglucinolysis (Hanlin \& Downey, 2009). However, because this approach includes removal of some oligomers, and because some terminal units would be degraded by phloroglucinolysis, average polymer length would also be overestimated. The removal of non-tannin and low molecular weight material by liquid-liquid separation prior to phloroglucinolysis substantially improves HPLC peak resolution and identification.

Unfortunately for this investigation, the method of liquidliquid purification normally used in our laboratories was optimised for the purification of tannin extracted in $70 \%$ acetone (Hanlin \& Downey, 2009) and proved to be incompatible with ethanol extraction solvents and extraction solvents with low concentrations of acetone. Thus, in this investigation, all of the tannin extracts were analysed by HPLC-phloroglucinolysis without further purification to maintain a consistent approach. Another approach would have been to use solid phase extraction, but dimethylcinnamaldehyde staining (Li et al., 1996) indicated that some tannin material was usually retained on these columns and this was not pursued further.

The solubility of flavan-3-ols also varies with different solvents, with monomers being more soluble in 50\% ethanol than in 70\% acetone (Kallithraka et al., 1995). The aqueous ethanol extracts of tannin may have higher concentrations of monomers, some of which were converted to degradation adducts. The lack of a reliable method warrants a certain amount of circumspection when analytical data is interpreted. The choice of extraction solvents and analytical methods can therefore influence the results obtained.

The tannins extracted in most of the solvents, particularly $50 \%$ ethanol and $70 \%$ acetone, had similar compositions. This suggests that there is most probably a single type of tannin present in wine grapes. The differences in total tannins observed by Seddon and Downey (2008) using different analytical methods could be partially due to solvent choice. Differences in the subunit composition of tannin polymers probably contribute little to the observed discrepancy. However, there were significant differences in average polymer length and polymer number and this can be the fundamental basis of the differences between the analytical 
methods (Harbertson \& Downey, 2009). The analytical methods employed by Seddon and Downey (2008) do not describe the polymer length distribution of the tannins in grape skin, although gel permeation chromatography adapted for HPLC (Kennedy \& Taylor, 2003) may be a suitable analytical tool for this purpose.

\section{CONCLUSIONS}

Acetone-water mixtures were more effective solvents than ethanolwater mixtures for the extraction of condensed tannins from grape skin, and were equally effective across a range of concentrations from 50 to $70 \%(\mathrm{v} / \mathrm{v})$. The most effective concentration of ethanol in water was $50 \%(\mathrm{v} / \mathrm{v})$. At these concentrations, terminal and extension subunit composition was comparable to ethanol and acetone extracts, although average polymer length and polymer number varied. Based on these observations, $50 \%$ ethanol in water would be a suitable substitute for $70 \%$ acetone in cases where organic solvents such as acetone are unacceptable (e.g. sensory evaluation of wine). The extraction variability reported between the solvents indicates that the selection of solvent mixture is important and depends on the objective of the analyses to be performed on the extracted tannin.

\section{LITERATURE CITED}

Alonso, E., Bouzeix, M. \& Revilla, E., 1991. Suitability of water/ethanol mixtures for the extraction of catechins and proanthocyanidins from Vitis vinifera seeds contained in a winery by-product. Seed Sci. Technol. 19(2), 545-552.

Downey, M.O., Harvey, J.S. \& Robinson, S.P., 2003. Analysis of tannins in seeds and skins of Shiraz grapes throughout berry development. Aust. J. Grape Wine Res. 9(1), 15-27.

Foo, L.Y. \& Karchesy, J.J., 1989. Procyanidin polymers of Douglas Fir bark: structure from degradation with phloroglucinol. Phytochemistry 28(11), 31853190 .

Gawel, R., 1998. Red wine astringency: a review. Aust. J. Grape Wine Res. 4, 74-95.

Gu, L., Kelm, M., Hammerstone, J.F., Beecher, G., Cunningham, D., Vannozzi, S. \& Prior, R.L., 2002. Fractionation of polymeric procyanidins from lowbush blueberry and quantification of procyanidins in selected foods with an optimized normal-phase HPLC-MS fluorescent detection method. J. Agric. Food Chem. 50(17), 4852-4860.

Guyot, S., Le Guernevé, C., Marnet, N. \& Drilleau, J-F., 1999. Methods for determining the degree of polymerisation of condensed tannins: a new 1H-NMR procedure applied to cider apple procyanidins. In: Gross, G. et al. (eds). Plant Polyphenols 2. Chemistry, Biology, Pharmacology, Ecology. Kluwer Academic/ Plenum Publishers, New York. pp. 211 - 222.

Hagerman, A.E., 1988. Extraction of tannin from fresh and preserved leaves. J. Ecol. 14(2), 453-461.

Hanlin, R.L. \& Downey, M.O., 2009. Condensed tannin accumulation and composition in skin of Shiraz and Cabernet Sauvignon grapes during berry development. Am. J. Enol. Vitic. 60(1), 13-23.

Hanlin, R.L., Hrmova, M.H., Harbertson, J.F. \& Downey, M.O., 2010. Review: Condensed tannin and grape cell wall interactions and their impact on tannin extractability into wine. Aust. J. Grape Wine Res. 16(1), 173-188.

Harbertson, J.F. \& Downey, M.O., 2009. Investigating differences in tannin levels determined by methylcellulose and protein precipitation. Am. J. Enol. Vitic. 60(2), 246-249.

Harbertson, J.F., Kennedy, J.A. \& Adams, D.O., 2002. Tannin in skins and seeds of Cabernet Sauvignon, Syrah, and Pinot noir during ripening. Am. J. Enol. Vitic. 53(1), 54-59.

Hazak, J.C., Harbetson, J.F., Adams, D.O., Lin, C.H. \& Ro, B.H., 2005. The phenolic components of grape berries in relation to wine composition. Acta Hort. $689,189-196$

Kallithraka, S., Garcia-Viguera, C., Bridle, P. \& Bakker, J., 1995. Survey of solvents for the extractions of grape seed phenolics. Phytochem. Anal. 6(5), 265267.

Kantz, K. \& Singleton, V.L., 1990. Isolation and determination of polymeric polyphenols using Sephadex LH-20 and analysis of grape tissue extracts. Am. J. Enol. Vitic. 41(3), 223-228.

Kelm, M.A., Johnson, J.C., Robbins, F.J., Hammerstone, J.F. \& Schmitz, H.H., 2006. High-performance liquid chromatography separation and purification of cacao (Theobroma cacao L.) procyanidins according to degree of polymerization using a diol stationary phase. J. Agric. Food Chem. 54(5), 1571-1576.

Kennedy, J.A. \& Jones, G.P., 2001. Analysis of proanthocyanidin cleavage products following acid-catalysis in the presence of excess phloroglucinol. J. Agric. Food Chem. 49(4), 1740-1746.

Kennedy, J.A. \& Taylor, A.W., 2003. Analysis of proanthocyanidins by highperformance gel permeation chromatography. J. Chromatogr. A. 995(1-2), 99107.

Li, Y.G., Tanner, G. \& Larkin, P., 1996. The DMACA-HCl protocol and the threshold proanthocyanidin content for bloat safety in forage legumes. J. Sci. Food Agric. 70(1), 89-101.

Maury, C., Sarni-Mamchado, P., Lefebvre, S., Cheynier, V. \& Moutounet, M., 2003. Influence of fining with plant proteins on proanthocyanidin composition of red wines. Am. J. Enol. Vitic. 54(2), 105-111.

Mercurio, M., Dambergs, R.G., Herderich, M.J. \& Smith, P.A., 2007. High throughput analysis of red wine and grape phenolics - adaptation and validation of methyl cellulose precipitable tannin assay and modified Somers color assay to a rapid 96 well plate format. J. Agric. Food Chem. 55(12), 4651-4657.

Monagas, M., Gómez-Cordovés, C., Bartolomé, B., Laureano, O. \& Ricardo da Silva, J.M., 2003. Monomeric, oligomeric, and polymeric flavan-3-ol composition of wines and grapes from Vitis vinifera L. cv. Graciano, Tempranillo, and Cabernet Sauvignon. J. Agric. Food Chem. 51(22), 6475-6481.

Pastor del Rio, J. \& Kennedy, J.A., 2006. Development of proanthocyanidins in Vitis vinifera L. cv. Pinot Noir grapes and extraction into wine. Am. J. Enol. Vitic. $57(2), 125-132$

Pekic, B., Kovac, V., Alonso, E. \& Revilla, E., 1998. Study of the extraction of proanthocyanidins from grape seeds. Food Chem. 16(1-2), 201-206.

Peng, Z., Hayasaka, Y., Iland, P.G., Sefton, M., Hoj, P. \& Waters, E.J., 2001. Quantitative analysis of procyanidins (tannins) from grape (Vitis vinifera) seeds by reverse phase high-performance liquid chromatography. J. Agric. Food Chem. 49(1), 26-31.

Sarneckis, C.J., Dambergs, R.G., Jones, P., Mercurio, M., Herderich, M.J. \& Smith, P.A., 2006. Quantification of condensed tannins by precipitation with methyl cellulose: development and validation of an optimised tool for grape and wine analysis. Aust. J. Grape Wine Res. 12(1), 1-11.

Seddon, T.J. \& Downey, M.O., 2008. Comparisons of analytical methods for the determination of condensed tannins in grape skins. Aust. J. Grape Wine Res. 14(1), 54-61.

Skogerson, K.J., Downey, M.O., Mazza, M. \& Boulton, R.B., 2007. Rapid determination of phenolic components in red wines from uv-visible spectra and the method of partial least squares. Am. J. Enol. Vitic. 58(3), 318-325. 